Research Article

\title{
Approximate Lie Symmetry Conditions of Autoparallels and Geodesics
}

\author{
Sameerah Jamal \\ School of Mathematics, University of the Witwatersrand, Johannesburg, South Africa \\ Correspondence should be addressed to Sameerah Jamal; sameerah.jamal@wits.ac.za
}

Received 21 August 2020; Accepted 14 October 2020; Published 28 October 2020

Academic Editor: Shi Liang Wu

Copyright (c) 2020 Sameerah Jamal. This is an open access article distributed under the Creative Commons Attribution License, which permits unrestricted use, distribution, and reproduction in any medium, provided the original work is properly cited.

\begin{abstract}
This paper is devoted to the study of approximate Lie point symmetries of general autoparallel systems. The significance of such systems is that they characterize the equations of motion of a Riemannian space under an affine parametrization. In particular, we formulate the first-order symmetry determining equations based on geometric requirements and stipulate that the underlying Riemannian space be approximate in nature. Lastly, we exemplify the results by application to some approximate wave-like manifolds.
\end{abstract}

\section{Introduction}

In a $n$-dimensional Riemannian space, it is a formidable task to compute the Lie point symmetries of any equation in that space, and this problem is only exacerbated if such an equation possesses a small perturbation. In most, if not all cases, this computation cannot be performed with the aid of software programs. It is therefore of great interest to devise an alternate route to the symmetry vectors. Indeed, one such approach that has proven effective is to encase the symmetry determining problem in a geometric setting $[1,2]$. The complexity of the computations then decreases dramatically.

In lieu of this, in this paper, we illustrate that the approximate symmetry conditions can be cast into a set of simple mathematical expressions of a geometric nature. Consider a $C^{\infty}$ manifold $M$ of dimension $n$, endowed with a symmetric connection (the connection needs not be symmetric in general). The connection defines an autoparallel system which is a family of curves or paths on the manifold and where the covariant derivative of the tangent to these curves is parallel to itself, that is,

$$
\nabla_{\dot{x}(t)} \dot{x}(t)=\phi(t) \dot{x}(t),
$$

where - denotes the derivative with respect to the parameter $t$ along the curves. In a local coordinate system, equation (1) is a system of second-order ordinary differential equations (linear in the highest derivative)

$$
\ddot{x}^{i}(t)+\Gamma_{j k}^{i}\left(x(t) \dot{x}^{j}(t) \dot{x}^{k}(t)=(t) \dot{x}^{i}(t)\right),
$$

where $\Gamma_{j k}^{i}=(1 / 2) g^{i l} g_{j l, k}+g_{k l, j}-g_{j k, l}$. An additional point to note is that if it vanishes, the autoparallel system is affinely parameterized with the so-called affine parameter. In this case, the autoparallels are the geodesics of the Riemannian space. Since the geodesics are dependent on the metric of the space, some far reaching results have been established in the context of Lie point symmetries. Studies by Aminova $[3,4]$ illustrated that Lie symmetries provide the projective algebra of a space if the Cartan parametrization of the geodesics is selected. Other notable research is that contained in $[5,6]$ and of course the related works by Katzin and Levine [7]. It is therefore judicious to expect that the approximate Lie symmetries of the system of geodesics of a perturbed metric will be closely related to the approximate collineations of the metric. We aim to apply the results of approximate Lie point symmetries to perturbative autoparallels and generalize it in an approximate Riemannian space. Essentially, we will establish a geometric way of dealing with the autoparallel symmetry problem for the first-order perturbative Riemannian spaces. In a series of papers, we have explored 
connections between geometry and perturbations and showed how the inherited perturbations affect geometric symmetry conditions of the induced partial differential equations [8-11]. We showed that if a Riemannian metric contains small perturbations, any partial differential equation constructed on such a space will inherit the perturbations. We show here that this extends to ordinary differential equations, in particular, autoparallel systems and its subclass of homogeneous ordinary differential equations. While many authors have studied approximate symmetries of geodesic equations, none have explored how to generalize the construction of these symmetries, hence the novelty of this work. Ultimately, we stipulate a set of simple geometric formulae that, when solved, provide the approximate symmetries for the approximate autoparallels and geodesic equations of motion.

The paper is organized as follows. In Section 2, we discuss some of the existing theories on approximate symmetries of differential equations based on the work [12]. Section 3 gives the approximate nature of autoparallel systems in our work. Section 4 is the main section that shows the derivation of the approximate Lie point symmetry conditions of autoparallel systems in general form. Lastly, in Section 5, we present some examples to showcase the applicability of our results. In Section 6, we conclude.

\section{Approximate Lie Symmetries}

In the text, we consider a first-order approximation denoted by the perturbation parameter. An approximate equation

$$
F(z, \varepsilon) \equiv F_{0}(z)+\varepsilon F_{1}(z) \approx 0, z=z^{1}, \cdots, z^{N}
$$

is approximately invariant with respect to the one-parameter approximate transformation group

$$
\bar{z}^{i} \approx h(z, \alpha, \varepsilon) \equiv h_{0}^{i}(z, \alpha)+\varepsilon h_{1}^{i}(z, \alpha), \quad i=1, \cdots, N,
$$

( $~ \alpha, \varepsilon$ " are two infinitesimal parameters) with the generator

$$
X=X_{0}+\varepsilon X_{1}+O\left(\varepsilon^{2}\right)
$$

if and only if

$$
\left(X_{0} F_{0}(z)+\varepsilon\left(X_{1} F_{0}(z)+X_{0} F_{1}(z)\right)\right)_{\text {equation (3) }}=O(\varepsilon) .
$$

The determining equation (6) can be written as follows:

$$
\begin{aligned}
X_{0} F_{0}(z) & =\lambda(z) F_{0}(z), \\
X_{1} F_{0}(z)+X_{0} F_{1}(z) & =\lambda(z) F_{1}(z) .
\end{aligned}
$$

The factor $\lambda(z)$ is determined by (7) and then substituted into (8), where the latter equation holds for $F_{0}(z)=0$. Alternatively, one may evaluate ( 7$)$ to obtain the exact symmetries, then find an auxiliary function $H$ by virtue of (7), (8), and (3), that is,

$$
H=\frac{1}{\varepsilon} X_{0}\left(F_{0}(z)+\varepsilon F_{1}(z)\right)_{F_{0}(z)+\varepsilon F_{1}(z)=0} .
$$

Thereafter, $X_{1}$ is calculated by solving the determining equation for deformations

$$
\left.X_{1} F_{0}(z)\right|_{F_{0}(z)=0}+H=0 \text {. }
$$

We remark that there exists a secondary approach to dealing with symmetries of differential equations that admit small perturbations, where dependent variables are first expanded in a perturbation series and thereafter re-substituted into a given equation [13].

\section{The Approximate Autoparallel System}

Below, we consider the approximate autoparallels, not necessarily under the affine parameterization, of a symmetric connection. The construction of an approximate autoparallel system requires an approximate metric perturbed to the first order (the perturbation order may be higher, but this will not be considered here). Hence, consider the manifold $M$ of dimension $n \geq 3$, endowed with a (pseudo) Riemannian metric $g_{i j}$. The metric is decomposed into a sum of an exact and an approximate metric, according to the perturbation parameter $\varepsilon$, viz,

$$
g_{i j}=\sigma_{i j}+\varepsilon \gamma_{i j}+O \varepsilon^{2}
$$

with inverse

$$
\bar{g}^{i j}=\bar{\sigma}^{i j}+\varepsilon \bar{\gamma}^{i j}+O\left(\varepsilon^{2}\right)
$$

Thus, in the autoparallel system equation (1), we let

$$
\Gamma_{j k}^{i}=\left(A_{j k}^{i}+\varepsilon B_{j k}^{i}+\varepsilon C_{j k}^{i}\right)
$$

where $A_{j k}^{i}=(1 / 2) \bar{\sigma}^{i l} \sigma_{j l, k}+\sigma_{k l, j}-\sigma_{j k, l}, \quad B_{j k}^{i}=(1 / 2) \bar{\gamma}^{i l}\left(\sigma_{j l, k}+\right.$ $\left.\sigma_{k l, j}-\sigma_{j k, l}\right)$, and $C_{j k}^{i}=(1 / 2) \bar{\sigma}^{i l}\left(\gamma_{j l, k}+\gamma_{k l, j}-\gamma_{j k, l}\right)$.

A perturbed autoparallel equation is then of the form (3), where the exact or unperturbed part is

$$
F_{0}=\ddot{x}^{i}(t)+A_{j k}^{i}(x(t)) \dot{x}^{j}(t) \dot{x}^{k}(t)-\phi(t) \dot{x}^{i}(t)
$$

and the approximate constituent is

$$
F_{1}=\left(B_{j k}^{i}(x(t))+C_{j k}^{i}(x(t))\right) \dot{x}^{j}(t) \dot{x}^{k}(t) .
$$

\section{Generalized Formulae for the Determining Conditions}

Next, we construct general symmetry conditions for the approximate Lie symmetries of equation (3) with (14) and 
(15). Hence, suppose that the symmetry generator (5) is of the form

$$
\begin{aligned}
& X_{0}=\xi_{0}(t, x, \dot{x}) \partial_{t}+\eta_{0}^{i}(t, x, \dot{x}) \partial_{x^{i}}, \\
& X_{1}=\xi_{1}(t, x, \dot{x}) \partial_{t}+\eta_{1}^{\mathrm{i}}(t, x, \dot{x}) \partial_{x^{i}} .
\end{aligned}
$$

The exact Lie point symmetries (16) of the equation (14) are found in the standard way. In fact, a detailed study of the exact symmetries of the autoparallels was completed in [14]. One applies condition (7),

$$
\begin{aligned}
X_{0} & \left(\ddot{x}^{i}(t)+A_{j k}^{i}(x(t)) \dot{x}^{j}(t) \dot{x}^{k}(t)-\phi(t) \dot{x}^{i}(t)\right) \\
& =\lambda\left(\ddot{x}^{i}(t)+A_{j k}^{i}(x(t)) \dot{x}^{j}(t) \dot{x}^{k}(t)-(t) \dot{x}^{i}(t)\right),
\end{aligned}
$$

where it is necessary to prolong $X_{0}$.

The first prolongation is

$$
X_{0}^{[1]}=X_{0}+\left(\frac{d}{d t} \eta_{0}^{i}-\dot{x}^{i} \frac{d}{d t} \xi_{0}\right) \partial_{\dot{x}^{i}}
$$

where

$$
\frac{d}{d t} \eta_{0}^{i}-\dot{x}^{i} \frac{d}{d t} \xi_{0}=\eta_{0, t}^{i}+\eta_{0, j}^{i} \dot{x}^{j}-\xi_{0, t} \dot{x}^{i}-\xi_{0, j} \dot{x}^{i} \dot{x}^{j}
$$

while a second-order prolongation is

$$
\begin{aligned}
& X_{0}^{[2]}=X_{0}^{[1]}+\left(\frac{d}{d t}\left(\dot{\eta}_{0}^{i}-\dot{x}^{i} \dot{\xi}_{0}\right)-\ddot{x}^{i} \frac{d}{d t} \xi_{0}\right) \partial_{\ddot{x}^{i}}, \\
& \frac{d}{d t}\left(\dot{\eta}_{0}^{i}-\dot{x}^{i} \dot{\xi}_{0}\right)-\ddot{x}^{i} \frac{d}{d t} \dot{\xi}_{0}= \dot{\eta}_{0, t t}^{i}+2 \dot{\eta}_{0, t j}^{i} \dot{x}^{j}+\dot{\eta}_{0, k j}^{i} \dot{x}^{k} \dot{x}^{j}-\dot{\xi}_{0, t t} \dot{x}^{i} \\
&-2 \dot{\xi}_{0, t j} \dot{x}^{j} \dot{x}^{i}-\dot{\xi}_{0, j k} \dot{x}^{j} \dot{x}^{i} \dot{x}^{k}+\dot{\eta}_{0, j}^{i} \ddot{x}^{j} \\
&-2 \dot{\xi}_{0, t} \ddot{x}^{i}-\dot{\xi}_{0, j} \dot{x}^{\dot{x}^{j}}-2 \dot{\xi}_{0, j} \ddot{x}^{i} \dot{x}^{j} .
\end{aligned}
$$

A solution of condition (18) provides the symmetry coefficients $\xi_{0}(t, x, \dot{x}), \eta_{0}^{i}(t, x, \dot{x})$ of $(16)$ and the factor

$$
\lambda=-\dot{\xi}_{0}
$$

It is then necessary to find the symmetry coefficients $\xi_{1}(t, x, \dot{x}), \eta_{1}^{i}(t, x, \dot{x})$ to obtain (17) explicitly.

The approximate Lie point symmetries of (3) are found by condition (8)

$$
\begin{aligned}
X_{0}( & \left.\left(B_{j k}^{i}(x(t))+C_{j k}^{i}(x(t))\right) \dot{x}^{j}(t) \dot{x}^{k}(t)\right) \\
& +X_{1}\left(\ddot{x}^{i}(t)+A_{j k}^{i}(x(t)) \dot{x}^{j}(t) \dot{x}^{k}(t)-\phi(t) \dot{x}^{i}(t)\right) \\
= & \lambda\left(\left(B_{j k}^{i}(x(t))+C_{j k}^{i}(x(t))\right) \dot{x}^{j}(t) \dot{x}(t)\right),
\end{aligned}
$$

where again it is necessary to prolong $X_{0}$ and $X_{1}$ as well. The prolongation formulae are analogous to (19) and (21), but with the "0" subscript replaced with " 1 ."

Omitting the substitution of the prolongation formulae into (24) and its subsequent lengthy expansion, at this stage, we collect all salient terms of the same order in $\dot{x}^{i}$ in equation (24) to find the following determining system for (17).

$(\dot{x})^{0}$ terms $: \eta_{1, t t}^{i}+\eta_{1, t}^{i} \phi+\eta_{0, t t}^{i}=0$,

$\left(\dot{x}^{1}\right)$ terms $: \xi_{0, t t} \delta_{j}^{i}-2\left(\eta_{0, t j}^{i}+\eta_{0, t}^{k} B_{(k j)}^{i}+\eta_{0, t}^{k} C_{(k j)}^{i}\right)$

$$
\begin{aligned}
& +\xi_{1, t t} \delta_{j}^{i}-\xi_{1} \phi_{, t} \delta_{j}^{i}-2\left(\eta_{1, t j}^{i}+\eta_{1, t}^{k} A_{(k j)}^{i}\right) \\
& -\left(\phi \xi_{1, t}+\phi_{, k} \eta_{1}^{k}\right) \delta_{j}^{i}=0,
\end{aligned}
$$

$$
\begin{aligned}
\left(\dot{x}^{2}\right) \text { terms : } & \left(-\eta_{0,(j k)}^{i}-\eta_{0}^{h} B_{(j k), h}^{i}-\eta_{0, k}^{h} B_{h j}^{i}-\eta_{0, k}^{h} B_{j h}^{i}+\eta_{0, h}^{i} B_{j k}^{h}\right) \\
& +2 \xi_{0, t\left({ }_{j} \delta_{k}^{i}\right)}-\xi_{0} B_{(k j), t}^{i}+\left(-\eta_{0}^{h} C_{(j k), h}^{i}-\eta_{0, k}^{h} C_{h j}^{i}\right. \\
& \left.-\eta_{0, k}^{h} C_{j h}^{i}+\eta_{0, h}^{i} C_{j k}^{h}\right)-\xi_{0} C_{(k j), t}^{i} \\
& +\left(-\eta_{1,(j k)}^{i}-\eta_{1}^{h} A_{(j k), h}^{i}-\eta_{1, k}^{h} A_{h j}^{i}\right. \\
& \left.\left.-\eta_{1, k}^{h} A_{j h}^{i}+\eta_{1, h}^{i} A_{j k}^{h}\right)+2 \xi_{1, t\left(j \delta_{k}\right.}^{i}\right) \\
& -2 \phi \xi_{1,\left(j \delta_{k} \delta_{k}^{i}\right)}-\xi_{1} A_{(k j), t}^{i}=0 .
\end{aligned}
$$

We may simplify the expression (27) to

$$
\begin{aligned}
L_{\eta_{1}} A_{(j k)}^{i}+L_{\eta_{0}} B_{(j k)}^{i}+L_{\eta_{0}} C_{(j k)}^{i}= & -2 \xi_{1, t(j} \delta_{k)}^{i}+2 \phi \xi_{1,(j} \delta_{k)}^{i} \\
& +\xi_{1} A_{(k j), t}^{i}-\eta_{0,(j k)}^{i} .
\end{aligned}
$$

$\left(\dot{x}^{3}\right)$ terms : $\left(\xi_{1,(j k}-\xi_{1,|s|} A_{j k}^{s}\right) \delta_{h)}^{i}-\xi_{0,|s|} B_{j k}^{s} \delta_{h)}^{i}-\xi_{0,|s|} C_{j k}^{s} \delta_{h)}^{i}=0$.

Once a specific metric is identified, the symmetry conditions (25)-(29) are solved to obtain $X_{1}$.

Finally, we remark that if one wanted the auxiliary function $H$, it is in a general form

$$
\begin{aligned}
H= & \varepsilon^{-1}\left[\left(\xi_{0} \partial_{t}+\eta_{0}^{i} \partial_{x^{i}}+\left(\eta_{0, t}^{i}+\eta_{0, j}^{i} \dot{x}^{j}-\xi_{0, t} \dot{x}^{i}-\xi_{0, j} \dot{x}^{i} \dot{x}^{j}\right) \partial_{\dot{x}^{i}}\right.\right. \\
& +\left(\eta_{0, t t}^{i}+2 \eta_{0, t j}^{i} \dot{x}^{j}+\eta_{0, k j}^{i} \dot{x}^{k} \dot{x}^{j}-\xi_{0, t t} \dot{x}^{i}-2 \xi_{0, t j} \dot{x}^{j} \dot{x}^{i}\right. \\
& \left.\left.-\xi_{0, j k} \dot{x}^{j} \dot{x}^{i} \dot{x}^{k}+\eta_{0, j}^{i} \ddot{x}^{j}-2 \xi_{0, t} \ddot{x}^{i}-\xi_{0, j} \dot{x}^{i} \ddot{x}^{j}-2 \xi_{0, j} \ddot{x}^{i} \dot{x}^{j}\right) \partial_{\dot{x}^{i}}\right) \\
& \left.\cdot\left(\ddot{x}^{i}(t)+\Gamma_{j k}^{i}(x(t)) \dot{x}^{j}(t) \dot{x}^{k}(t)-\phi(t) \dot{x}^{i}(t)\right)\right], \\
& \bmod F_{0}+F_{1}=0 .
\end{aligned}
$$

An important conclusion here is that the approximate Lie symmetry vector of perturbed spaces is easily found from the 
solution of geometric equations. Next, we demonstrate the application of the results in various important cases.

\section{Applications: The Affine Space}

The most valuable application of the above formulae is the affine parametrization with $\phi(t)=0$, that is, the equations of motion. We compute the approximate Lie symmetry vectors for the case of affine parametrization and the assumption that $\Gamma_{j k, t}^{i}=0$, since the metric does not depend on the affine parameter.

It is well known that the exact symmetries for geodesic equations are limited to the following form:

$$
X_{0}=\left(R^{k}(t) S^{k}(x)+T(t)\right) \partial_{t}+\left(P^{i}(x) t+Q^{i}(x)\right) \partial_{x^{i}}
$$

$P^{i}(x), Q^{i}(x)$ are arbitrary differentiable vector fields, $R^{k}(t), T(t)$ are arbitrary functions of the affine parameter $t$, and $S^{k}(x)$ is an arbitrary function, but further details can be mentioned if one makes assumptions about whether the metric admits gradient Killing vectors or not [14]. Additionally, the $R^{J}(t) S^{J}(x)+T(t)$ is at most a function of $t^{2}$. The exact symmetry vectors in each case considered below are easily identifiable. On the other hand, the approximate vectors are more involved but their determination is facilitated by the symmetry determining conditions established above.

5.1. Case A: Perturbed Cylindrically Symmetric Static SpaceTime. A perturbative cylindrically symmetric static metric is [15]

$$
\begin{aligned}
d s^{2}= & e^{\rho^{2} / R^{2}} d t^{2}-d \rho^{2}-e^{\rho^{3} / R^{3}}\left(p^{2} d \phi^{2}+d z^{2}\right) \\
& +\frac{2 t \varepsilon}{T}\left(e^{\rho^{2} / R^{2}} d t^{2}-e^{\rho^{3} / R^{3}}\left(d \phi^{2}+d z^{2}\right)\right),
\end{aligned}
$$

where $p$ is a constant and $R$ is a constant of dimensions of $\rho$.

The approximate geodesic equations of the form (3) are:

$$
\begin{aligned}
\ddot{z}= & -\frac{\dot{z}}{R^{3} T}\left(2 R^{3} \varepsilon \dot{t}+3 T x^{2} \dot{x}\right), \\
\ddot{t}= & \frac{1}{R^{2} T}\left(e^{\rho^{3} / R^{3}} R^{2} \varepsilon p^{2} \dot{\phi}^{2}+\varepsilon e^{\rho^{2} / R^{2}} \dot{t}^{2} R^{2}+e^{\rho^{3} / R^{3}} R^{2} \varepsilon \dot{z}^{2}\right. \\
& \left.+2 e^{\rho^{2} / R^{2}} T \dot{t} \rho \dot{\rho}\right)\left(e^{-\rho^{2} / R^{2}}\right), \\
\ddot{\rho}= & \frac{1}{2} \frac{\rho}{R^{3} T}\left(-6 e^{\rho^{3} / R^{3}} \varepsilon p^{2} t \rho \dot{\phi}^{2}-3 e^{\rho^{3} / R^{3}} T p^{2} \rho \dot{\phi}^{2}+4 e^{\rho^{2} / R^{2}} a \varepsilon t \dot{t}^{2}\right. \\
& \left.-6 e^{\rho^{3} / R^{3}} \varepsilon t \rho \dot{z}^{2}+2 e^{\rho^{2} / R^{2}} T R \dot{t}^{2}-3 e^{\rho^{3} / R^{3}} T \rho \dot{z}^{2}\right), \\
\ddot{\phi}= & \frac{\dot{\phi}}{R^{3} T}\left(2 R^{3} \varepsilon \dot{t}+3 T x^{2} \dot{x}\right) .
\end{aligned}
$$

Application of (25)-(29) and their solution provides the following 7 (exact and approximate) symmetries:

$$
\begin{aligned}
& X^{1}=\partial_{s}, \\
& X^{2}=s \partial_{s}, \\
& X^{3}=\varepsilon \partial_{t}, \\
& X^{4}=\partial_{\phi}, \\
& X^{5}=\partial_{z}, \\
& X^{6}=-z \partial_{\phi}+p^{2} \phi \partial_{z}, \\
& X^{7}=2 \partial_{t}-\frac{\varepsilon}{T^{2} R^{3}} t \partial_{t}+\phi \partial_{\phi}+z \partial_{z},
\end{aligned}
$$

where, here, $s$ denotes the affine parameter.

5.2. Case B: Perturbed Plane Symmetric Statics Pace-Time. The metric in this case is

$$
\begin{aligned}
d s^{2}= & e^{2 x / a} d t^{2}-d x^{2}-e^{2 x^{2} / a^{2}}\left(d y^{2}+d z^{2}\right) \\
& +\frac{2 t \epsilon}{T}\left(e^{2 x / a} d t^{2}-e^{2 x^{2} / a^{2}}\left(d y^{2}+d z^{2}\right)\right),
\end{aligned}
$$

where $a$ is a constant and $T$ is a constant of dimensions of $t[16]$.

The approximate geodesic equation (1) is

$$
\begin{aligned}
\ddot{x}= & -\frac{\dot{t}^{2}}{a} e^{2(x / a)}+2 \frac{x \dot{y}^{2}}{a^{2}} e^{2\left(x^{2} / a^{2}\right)} \\
& -\frac{\varepsilon t}{T}\left(2 \frac{\dot{t}^{2}}{a} e^{2(x / a)}-4 \frac{x \dot{y}^{2}}{a^{2}} e^{2\left(x^{2} / a^{2}\right)}\right), \\
\ddot{y}= & -\frac{1}{2} e^{-2\left(x^{2} / a^{2}\right)}\left(4 \frac{\varepsilon \dot{y} \dot{t}}{T} e^{2\left(x^{2} / a^{2}\right)}+8 \frac{x \dot{y} \dot{x}}{a^{2}} e^{2\left(x^{2} / a^{2}\right)}\right), \\
\ddot{z}= & -\frac{1}{2} e^{-2\left(x^{2} / a^{2}\right)}\left(4 \frac{\varepsilon \dot{z} \dot{t}}{T} e^{2\left(x^{2} / a^{2}\right)}+8 \frac{x \dot{z} \dot{x}}{a^{2}} e^{2\left(x^{2} / a^{2}\right)}\right), \\
\ddot{t}= & \frac{1}{2} e^{-2(x / a)}\left(2 \frac{\varepsilon}{T}\left(e^{2(x / a)} \dot{t}^{2}-e^{2\left(x^{2} / a^{2}\right)} \dot{y}^{2}\right)\right. \\
& \left.-4 \frac{\varepsilon \dot{t}^{2}}{T} e^{2(x / a)}-4 \frac{\dot{t} \dot{x}}{a} e^{2(x / a)}\right) .
\end{aligned}
$$

The solution of conditions (25)-(29) provide the following 7 (exact and approximate) symmetries

$$
\begin{aligned}
& Y^{1}=\partial_{y}, \\
& Y^{2}=X^{1}, \\
& Y^{3}=X^{2}, \\
& Y^{4}=X^{3},
\end{aligned}
$$




$$
\begin{aligned}
& Y^{5}=\partial_{z}, \\
& Y^{6}=y \partial_{z}-z \partial_{y}, \\
& Y^{7}=\partial_{t}-\frac{\varepsilon}{T^{2} a^{2}} t \partial_{t}+y \partial_{y}+z \partial_{z} .
\end{aligned}
$$

\section{Concluding Remarks}

The objective of this work was to formalize the computation of approximate Lie point symmetries of autoparallel systems under a generic Riemannian space up to the first order in the perturbation parameter $\varepsilon$. To facilitate this, we set up explicit geometric equations that, when solved, provide the approximate symmetries of the autoparallel systems. These equations can be extended to the affinely parametrized geodesics which are a special case of the autoparallel system. We note that the application of equations (25)-(29) to the existing results in the literature [17-19] provides consistent results. To showcase the applicability of our formulae, we considered specific perturbed metrics and the symmetry conditions were applied to the geodesics of an approximate Riemannian space to find the approximate Lie point symmetry vectors. It is also possible to extend this analysis to the case of Noether symmetries for the Lagrangian function of the geodesic equation [20] and also the case of quadratic symmetries [21]. In a forthcoming work, we shall explore the higher-order approximate symmetries of the geodesic Lagrangian function.

\section{Data Availability}

All results are produced by the author.

\section{Conflicts of Interest}

The author declares no conflict of interest.

\section{Acknowledgments}

This study has received financial support from the National Research Foundation of South Africa (99279).

\section{References}

[1] S. Jamal and N. Mnguni, "Approximate conditions admitted by classes of the Lagrangian $\mathscr{L}=1 / 2\left(-u^{\prime 2}+u^{2}\right)+\epsilon^{i} G_{i}\left(u, u^{\prime}\right.$, $\left.u^{\prime \prime}\right)$," Applied Mathematics and Computation, vol. 335, pp. 65-74, 2018.

[2] A. Paliathanasis and S. Jamal, "approximate Noether symmetries and collineations for regular perturbative Lagrangians," Journal of Geometry and Physics, vol. 124, pp. 300-310, 2018.

[3] A. V. Aminova, "Automorphisms of geometric structures as symmetries of differential equations (in Russian)," Izvestiya Vysshikh Uchebnykh Zavedenii Matematika, vol. 2, pp. 3-10, 1994.

[4] A. V. Aminova, "Projective transformations and symmetries of differential equation," Sbornik: Mathematics, vol. 186, no. 12, pp. 1711-1726, 1995.

[5] T. Feroze, F. M. Mahomed, and A. Qadir, "The connection between isometries and symmetries of geodesic equations of the underlying spaces," Nonlinear Dynamics, vol. 45, no. 1-2, pp. 65-74, 2006.

[6] G. E. Prince and M. Crampin, "Projective differential geometry and geodesic conservation laws in general relativity. I: projective actions," General Relativity and Gravitation, vol. 16, no. 10, pp. 921-942, 1984.

[7] G. H. Katzin and J. Levine, "Geodesic first integrals with explicit path-parameter dependence in Riemannian spacetimes," Journal of Mathematical Physics, vol. 22, no. 9, pp. 1878-1891, 1981.

[8] S. Jamal, "nth-Order approximate Lagrangians induced by perturbative geometries," Mathematical Physics, Analysis and Geometry, vol. 21, no. 3, p. 25, 2018.

[9] S. Jamal, "Approximate conservation laws of nonvariational differential equations," Mathematics, vol. 7, no. 7, p. 574, 2019.

[10] S. Jamal, "Perturbative manifolds and the Noether generators of nth-order Poisson equations," Journal of Differential Equations, vol. 266, no. 7, pp. 4018-4026, 2019.

[11] S. Jamal, "Geometrization of heat conduction in perturbative space-times," Canadian Journal of Physics, vol. 97, no. 2, pp. 187-191, 2019.

[12] V. A. Baikov, R. K. Gazizov, and N. H. Ibragimov, "Approximate symmetries of equations with a small parameter," Matematicheski_i, Sbornik, vol. 136, pp. 435-450, 1988, (English Transl. in Math. USSR Sb. 64 (1989) 427).

[13] W. I. Fushchych and W. H. Shtelen, "On approximate symmetry and approximate solution of the non-linear wave equation with a small parameter," Journal of Physics A: Mathematical and General, vol. 22, p. 887, 1989.

[14] M. Tsamparlis and A. Paliathanasis, "Lie symmetries of geodesic equations and projective collineations," Nonlinear Dynamics, vol. 62, no. 1-2, pp. 203-214, 2010.

[15] A. Qadir and I. Hussain, "Approximate Symmetries and the Energy in Spacetimes," in The 12th Marcel Grossmann Meeting, pp. 1868-1873, World Scientific, 2012.

[16] I. Hussain and A. Qadir, "Approximate Noether symmetries of Lagrangian for plane symmetric gravitational wave-like spacetimes," Il Nuovo Cimento B, vol. 122, pp. 593-597, 2007.

[17] U. Camci, "Approximate Noether gauge symmetries of the Bardeen model," European Physical Journal C: Particles and Fields, vol. 74, no. 12, p. 3201, 2014.

[18] A. H. Kara, F. M. Mahomed, and A. Qadir, "Approximate symmetries and conservation laws of the geodesic equations for the Schwarzschild metric," Nonlinear Dynamics, vol. 51, pp. 183-188, 2008.

[19] M. Sharif and S. Waheed, "Energy content of colliding plane waves using approximate Noether symmetries," Brazilian Journal of Physics, vol. 42, no. 3-4, pp. 219-226, 2012.

[20] M. Tsamparlis and A. Paliathanasis, "The geometric nature of Lie and Noether symmetries," General Relativity and Gravitation, vol. 43, no. 6, pp. 1861-1881, 2011.

[21] L. Karpathopoulos, M. Tsamparlis, and A. Paliathanasis, "Quadratic conservation laws and collineations: a discussion," Journal of Geometry and Physics, vol. 133, pp. 279-286, 2018. 